\title{
Physikalische Therapie: Therapiemittel Elektrizität
}

\author{
Jean-Michel Jeannin
}

Basel, Schweiz

\section{Einleitung und physikalische Grundlagen}

In der Elektrotherapie werden unterschiedliche Stromformen medizinisch angewendet. Hierzu wird der elektrische Strom über Elektroden in das Gewebe geleitet. Gleichstrom kann auch durch Wasserbäder in den Körper geführt werden. Die Elektrotherapie erlaubt es, direkt am Membranpotenzial einer Zelle einzugreifen, d.h. dort, wo elektrische Signale entstehen und vermittelt werden. Solche direkten Wirkungen auf die Nervenfunktion können keine anderen Methoden der physikalischen Therapie leisten [1].

\section{Anwendungstechnik}

Der elektrische Strom wird entweder mittels Elektroden oder mittels Wasserbad auf den Patienten übertragen. Seine Wirksamkeit hängt von der Stromdichte (in mA/ $\mathrm{cm}^{2}$ ) an den Elektroden ab (Kasten). Die Dosierung erfolgt nach der subjektiven Empfindlichkeit. An der Sensibilitätsschwelle ist der Strom gerade wahrnehmbar, darunter gerade nicht wahrnehmbar und über der Sensibilitätsschwelle deutlich, aber nicht schmerzhaft wahrnehmbar [1]. Der zur Behandlung angewendete Strom (Abb. 1, 2) wird in folgende Frequenzbereiche eingeteilt: Niederfrequenzstrom $(0-1000 \mathrm{~Hz})$, Mittelfrequenzstrom $(1000-100000 \mathrm{~Hz})$ und Hochfrequenzstrom $(>100000 \mathrm{~Hz})$ [1].

\section{Niederfrequenztherapie}

Die häufigsten für die Niederfrequenztherapie angewendeten Stromformen sind diadynamische Ströme, Rechteckströme (Ultrareizstrom nach Träbert) $[1,2]$ und weitere Strommodalitäten für die Transkutane Elektrische Nervenstimulation (TENS). Die Reizstromtherapie
Kasten. Elektrischer Strom

Elektrischer «Strom» bedeutet den Fluss von Elektronen bzw. Ionen. Die Stromstärke wird in Ampere (A) angegeben. Für die therapeutische Anwendung reichen Stromstärken im Bereich von wenigen Milliampere (mA) aus.

Der Stromfluss wird von der Spannung erzeugt, die in Volt (V) angegeben wird.

Ein Stromleiter behindert den Stromfluss; die Behinderung wird als elektrischer Widerstand bezeichnet. Seine Einheit ist das Ohm (W). Am meisten Widerstand leisten die Haut, das subkutane Fettgewebe und die Knochen. Der Hautwiderstand ist in Abhängigkeit von der Hautdicke und -feuchtigkeit, vom Stoffwechsel und von der Durchblutung variabel [2]. Elektrischer Strom, der seine Richtung (Polarität) periodisch ändert, heisst Wechselstrom. Die Häufigkeit des Wechsels wird in $\mathrm{Hertz}(\mathrm{Hz})$ angegeben [1].

Gleichstrom ändert die Polarität nicht [1].

nach Träbert ist vor allem zur Behandlung von schmerzhaft verspannter Muskulatur geeignet [1]. Die in einer Reiztherapie am häufigsten angewendeten Stromformen sind die diadynamischen Ströme: MF (monophasé fixe, $50 \mathrm{~Hz}$ ); DF (diphasé fixe, $100 \mathrm{~Hz}$ ); CP (courtes périodes, rascher Wechsel von MF und DF); LP (longues périodes, langsamer Wechsel von MF und DF). MF wirkt stärker motorisch und sensibel stimulierend, CP wirkt stärker resorptionsfördernd, DF wirkt vorherrschend analgetisch und LP ist überwiegend detonisierend [1]. Diadynamische Ströme werden im Rahmen einer Reiztherapie zur Analgesie und zur Förderung der Resorption nach stumpfen Verletzungen angewendet. Die Gleichstromtherapie (Galvanisierung) wird überwiegend in hydrogalvanischen Voll- und Teilbädern angewendet. Sowohl im Vollbad («Stangerbad») als auch im Teilbad gibt es die Möglichkeit von variablen Schaltungen. Im Teilbad entfallen die Wirkungen der Immersion. Galvanisierung wirkt analgetisch, sedierend und erregungshemmend. In einer Iontophorese wird Gleichstrom zum Transport von ionisierten Wirkstoffen (z.B. Lokalanästethika, topische Antirheumatika, Histamin) in und durch die Haut angewendet. Dazu wird

\section{KARGER}

Fax +497614520714 Information@Karger.com www.karger.com
(C) 2016 S. Karger GmbH, Freiburg

$1015-0684 / 16 / 0283-0154 \$ 39.50 / 0$ 
Abb. 1. Kraftwerk Birsfelden (BL).

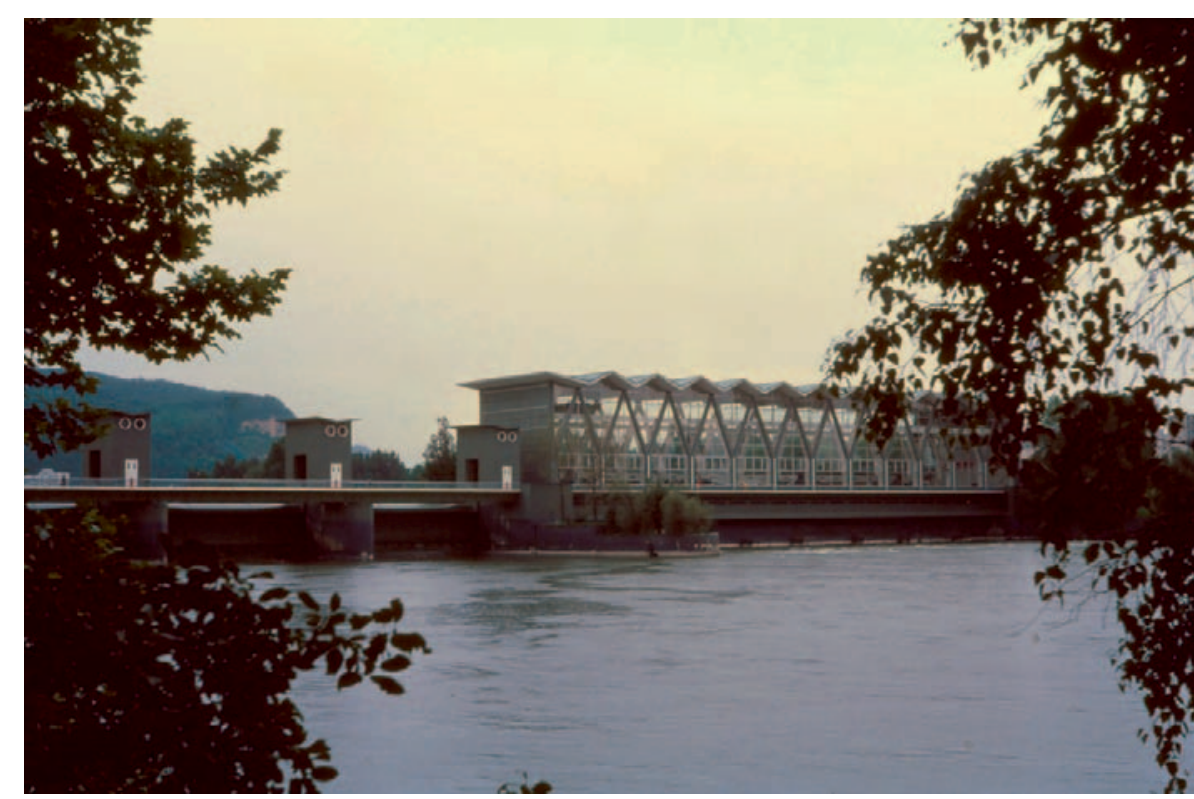

der Wirkstoff auf die gleichsinnig geladene Elektrode aufgetragen (positiv geladene Substanzen an Kathode, negativ geladene Substanzen an Anode) [1].

\section{Mittelfrequenztherapie}

In einer Mittelfrequenztherapie werden in der Regel Interferenzströme verwendet. Dazu wird das zu behandelnde Gebiet mittels zwei getrennter Stromkreise zwei sinusförmigen mittelfrequenten Strömen ausgesetzt. Die Elektroden werden über Kreuz geschaltet und so angelegt, dass sich die beiden Ströme im behandelten Gebiet überlagern. Im Bereich von 25-50 Hz übt der Strom eine Reizwirkung auf die Muskulatur aus. Im Bereich 90-100 Hz wirkt er sympatholytisch und analgetisch. Die Mittelfrequenztherapie wird auch zur Behandlung der Psoriasis angewendet $[3,4]$.

\section{Hochfrequenztherapie}

Im Unterschied zur Mittelfrequenz- und Niederfrequenztherapie werden mit einer Hochfrequenztherapie keine Aktionspotenziale und Muskelkontraktionen ausgelöst. Die Behandlung mit hochfrequentem Strom erzeugt im Körper Wärme. Somit ist sie eine Wärmebehandlung (Diathermie). Am häufigsten wird die Kurzwelle $(27 \mathrm{MHz})$ angewendet. Das Behandlungsgebiet befindet sich zwischen zwei Spulen oder zwei Kondensatorplatten. Die Hochfrequenztherapie wird zur Behandlung von schmerzhaften Zuständen des Bewegungsapparats und bei chronischen Entzündungen innerer Organe (Adnexitis, Sinusitis) verwendet [1].



Abb. 2. Turbine im Maschinenhaus des Kraftwerks Birsfelden.

\section{Wirkungsmechanismen}

Die Wirkung von elektrischem Strom setzt an den Nerven- und Muskelzellen an. Diese weisen an ihrer Membran ein elektrisches Potenzial von rund $-90 \mathrm{mV}$ (innen gegen aussen) auf. Wirkt ein Reiz auf die Membran, wird sie leicht depolarisiert. Ist der Reiz gross genug und die Wirkungszeit kurz genug $(<0,1 \mathrm{~ms})$, erfolgt eine vollständige Depolarisation, die dann fortgeleitet wird [5]. In der Elektrotherapie besteht der Reiz aus elektrischem Strom. Mit der Stromstärke, der Frequenz des Wechselstroms, der Dauer und der Platzierung der Elektroden kann der Reiz sehr fein reguliert werden. Sehr häufig wird die TENS angewendet. Folgende mögliche Mechanismen auf anatomisch-biochemischer Ebene werden diskutiert: präsynaptische Hemmung des Hinterhorns des Rückenmarks, das sensible Reize von der Peripherie an das Gehirn leitet, endogene Schmerzsteuerung via Endorphine, Enkephali- 
ne und Dynorphine, direkte Hemmung von abnormal erregten Nerven, Wiederherstellung von Afferenzen (sensible Reize, die von der Peripherie zum Gehirn geleitet werden) [6]. Die Wirkung bei der Behandlung der Psoriasis beruht möglicherweise auf einer Erhöhung von zyklischem Adenosinmonophosphat (AMP) [4], einer endogenen Substanz, die an der Signalübermittlung beteiligt ist.

\section{Ausgewählte Beispiele der klinischen Anwendung}

Philipp et al. [3] behandelten 12 Patienten mit einer Psoriasis der Handfläche mit Interferenzstrom im Rahmen einer älteren klinischen Studie. Als Kontrolle diente die spontane Remissionsrate. Nach 12 Wochen waren die Rötung, Schuppenbildung und Verhärtung sowie die Pusteln vermindert [3]. In einer neueren Prospektivstudie mit 9 Patienten mit psoriatrischer Arthritis beobachteten die Autoren eine Besserung der Schmerzhaftigkeit, jedoch keine Verbesserung bzw. sogar eine Verschlechterung des klinischen Befunds [4]. Eksioglu et al. [7] behandelten 50 Patienten, die an einer Fibromyalgie litten, entweder mit dem Antidepressivum Amitriptylin und einem täglichen Stangerbad oder mit Amitriptylin allein. Die mit Stangerbädern behandelten Patienten zeigten gegenüber den $\mathrm{Pa}$ tienten, die nur Amitriptylin erhielten, eine signifikante Verbesserung des «Fibromyalgia Impact Questionnaire (FIQ)»-Scores, wohingegen die Anzahl der gezählten Triggerpunkte in beiden Gruppen gleich blieb. Die Autoren kommen zu dem Schluss, dass die zusätzlichen Stangerbäder einen anhaltenden analgetischen Effekt haben und somit eine sinnvolle Massnahme darstellen [7]. In einer Übersichtsarbeit beurteilten DeSantana et al. [8] die Wirksamkeit einer TENS-Therapie bei schmerzhaften Zuständen des Bewegungsapparats als gegeben. Sie iden- tifizierten die adäquate Dosierung, d.h. die Intensität des Stroms, als kritischen Faktor für den Erfolg einer Behandlung [8].

\section{Risiken und Kontraindikationen}

Wechselstrom im Bereich der Netzfrequenz $(50 \mathrm{~Hz})$ ist ab einer Stromstärke von $0,5 \mathrm{~mA}$ spürbar. Stromstärken über $10 \mathrm{~mA}$, welche länger als $2 \mathrm{~s}$ einwirken, sind gefährlich. Gleichströme sind ab $2 \mathrm{~mA}$ spürbar und ab $25 \mathrm{~mA}$ gefährlich, wenn sie länger als 2 s einwirken [7]. Die Elektroden dürfen nicht direkt mit der Haut in Kontakt sein, weil die Gefahr von Verätzungen durch die Konzentration von Säuren und Laugen besteht [1]. Die wichtigsten Kontraindikationen sind Sensibilitätsstörungen, akute Entzündungen, aktive Phasen von degenerativen Gelenkveränderungen, Fieber, Infektionskrankheiten, Herzschrittmacher, Schwangerschaft (im Fall einer Anwendung im Abdominal- und Lumbalbereich) sowie Stromangst.

\section{Schlussbemerkungen}

Die Elektrotherapie ist eine typische Vertreterin der physikalischen Therapie. Ihre Wirksamkeit kann nicht ohne Weiteres gegenüber Placebo nachgewiesen werden, da die Patienten die Anwendung des elektrischen Stroms spüren. In einer der hier vorgestellten Studien wurde die Wirksamkeit mit der Spontanheilung verglichen; in einer anderen Studie wurde die Elektrotherapie (Stangerbad) zusätzlich zu einer medikamentösen Therapie verabreicht und gegenüber der Behandlung mit der medikamentösen Behandlung allein verglichen. Dem Autor erscheinen dies intelligente Alternativen zur placebogesteuerten Doppelblindstudie zu sein.

\section{Literatur}

1 Glaesener JJ: 3.7 Elektro- und Ultraschalltherapie; in Gutenbrunner C, Glaesener JJ: Rehabilitation, Physikalische Medizin und Heilverfahren. Heidelberg, Springer, 2007, p 69

2 From theory to practice; in den Adel RV, Luykx RHJ: Low and Medium Frequency Electrotherapy. Rotterdam, Enraf-Nonius, 2005, pp 12-23.

3 Philipp A, et al: Interferential current is effective in palmar psoriasis: an open prospective trial. Eur J Dermatol 2000;10:195-198.
Walker UA, et al: Analgesic and disease modifying effects of interferential current in psoriatic arthritis. Rheumatol Int 2006;26:904907.

5 Peripherer Nerv; in Keidel WD (Hrsg): Kurzgefasstes Lehrbuch der Physiologie, ed 1. Stuttgart, Thieme, 1969.

6 Kaye V: Transcutaneous Electrical Nerve Stimulation. http://emedicine.medscape.com/ article/325107-overview (aufgerufen am 14. April 2016).
Eksioglu E, et al: Effects of Stanger bath the rapy on fibromyalgia. Clin Rheumatol 2007; 26:691-694.

8 DeSantana JM, et al: Effectiveness of transcutaneous electrical nerve stimulation for treatment of hyperalgesia and pain. Curr Rheumatol Rep 2008;10:492-499.

9 Elektrischer Strom. Wikipedia: https:// de.wikipedia.org/wiki/Elektrischer_Strom (aufgerufen am 14. April 2016). 